\title{
On Localized Deformation and Recrystallization as Damage Mechanisms during Thermomechanical Fatigue of Single Crystal Nickel-Based Superalloys
}

\author{
Johan J. Moverare ${ }^{1,3, a}$, Atsushi Sato ${ }^{2, b}$, Sten Johansson ${ }^{1, c}$, \\ Magnus Hasselqvist ${ }^{3, \mathrm{~d}}$, Roger C. Reed ${ }^{2, \mathrm{e}}$, Jan Kanesund ${ }^{1, \mathrm{f}}$ \\ and Kjell Simonsson ${ }^{1, g}$ \\ ${ }^{1}$ Linköping University, Dep. of Management and Engineering, SE-581 83 Linköping, Sweden \\ ${ }^{2}$ University of Birmingham, Edgbaston, Birmingham, B15 2TT, UK \\ ${ }^{3}$ Siemens Industrial Turbomachinery $A B, 61283$ Finspång, Sweden \\ ajohan.moverare@liu.se, bXX5756@bham.ac.uk, 'sten.johansson@liu.se, \\ magnus.hasselqvist@siemens.com, r.c.reed@bham.ac.uk, fjan.kanesund@liu.se, \\ ${ }^{9}$ kjell.simonsson@liu.se
}

Key words: Thermomechanical fatigue (TMF), Single Crystals, Damage, Recrystallization

\begin{abstract}
Thermomechanical fatigue (TMF) in superalloys is growing in importance due to the introduction of advanced cooling systems but also due to the changes in demand and competition within the power generation market; this is requiring many power plants to operate under cyclic conditions. In this paper the TMF behaviour of three different single crystal nickel-based superalloys are compared. It is demonstrated that the deformation and damage mechanisms occurring during TMF are rather different from those traditionally reported for creep or isothermal fatigue. In all cases examined, the deformation is localized within a rather small number of deformation bands. While these bands were found to consist mainly of micro-twins in some alloys, in others they might be better described as slip or shear bands. Furthermore, in some circumstances these bands are prone to recrystallization. In CMSX-4, the intersection points of twins of different orientation act as initiation sites for this process. In the SCA425 alloy - of smaller gamma' content, lower creep resistance and less great oxidation resistance - twinning is observed infrequently; however the deformation is still very localized and in the distorted gamma-gamma' microstructure, along the shear bands, recrystallization is observed. Furthermore the recrystallization is enhanced by oxidation due to the development of a gamma'-depleted zone. In CMSX-4, TCP phases precipitated during long term ageing cause a more dispersed deformation behaviour which prevents recrystallization. Our findings confirm the importance of an inhomogeneous microstructure for good TMF resistance.
\end{abstract}

\section{Introduction}

The aim of the present paper is to summarize our recent efforts to characterize the deformation and damage mechanisms active during thermomechanical fatigue of single crystal Ni-base superalloys. Broadly speaking, thermomechanical fatigue (TMF) failure is promoted when plastic strains cannot be accommodated at low temperatures and creep deformation and/or oxidation occurs at high temperatures. Microstructural stability plays an important role during TMF and it is now established that there are important interactions between the degradation mechanisms occurring at high and low temperatures [1]. Therefore, the deformation and damage mechanisms in single crystal superalloys during TMF are very different from those traditionally reported to occur under creep or isothermal fatigue. Unfortunately, at this stage relatively little is known about the influence of alloy composition on the mechanisms active during thermomechanical fatigue of single crystal superalloys. In this study, the TMF performance of different alloys has been compared in order to reveal new insights into this topic. 


\section{Experimental details}

Siemens Industrial Turbomachinery $\mathrm{AB}$ has so far generated thermomechanical fatigue (TMF) data for more than 20 different Ni-base superalloys. In this study, attention is focussed on three monocrystalline alloys and the deformation and damage mechanisms seen in them during TMF. Some of the alloys have also been investigated in different heat treatment conditions, as indicated in Table 1. For further details regarding the alloys see references [2-6].

Table 1: Investigated alloys and heat treatment conditions

\begin{tabular}{|l|l|l|}
\hline Alloy & Heat treatment condition & Reference for further information \\
\hline CMSX-4 & Virgin condition: & {$[2],[3]$} \\
\hline & Long term aged: & {$[2],[3]$} \\
\hline SCA425Hf & Standard & {$[4]$} \\
\hline SCA425+ & HT A - Primary $\gamma^{\prime}$ size: $0.65 \mu \mathrm{m}$ & {$[5],[6]$} \\
\hline & HT B - Primary $\gamma^{\prime}$ size: $0.35 \mu \mathrm{m}$ & {$[5],[6]$} \\
\hline & HT C - Bi-modal & {$[5],[6]$} \\
\hline
\end{tabular}

Out of phase (OP) thermomechanical fatigue tests were conducted under mechanical strain control in the $100-950^{\circ} \mathrm{C}$ or $100-1000^{\circ} \mathrm{C}$ temperature range with a strain ratio of $\mathrm{R}=\varepsilon_{\min } / \varepsilon_{\max }=$ $\infty$. An MTS 810 servo-hydraulic thermomechanical fatigue machine with the MTS model 793 software was used for this purpose. In order to achieve a stabilized mean stress early in the tests a 20 hour hold time was applied at the maximum temperature $\left(\mathrm{T}_{\max }\right)$ during the first cycle, while all subsequent cycles had a 5 minute hold time. The combination of R-ratio and longer hold time in the first cycle is believed to better represent the real situation for most engineering components under OP-TMF loading. The single crystal alloys were tested along the [001] direction.

After testing to failure, the ruptured fatigue specimens were sectioned and prepared for microstructural investigations. Sectioning was performed both perpendicular and parallel to the longitudinal axis as well as parallel to the fracture surface, which in most cases was parallel to the $\{111\}$ planes. The samples were prepared by grinding and mechanical polishing. They were analysed subsequently using a Hitachi SU70 scanning electron microscope operating at $20 \mathrm{kV}$. No etching was performed on the samples and the contrast was achieved from differences in composition and crystallographic orientation only. Chemical composition information was obtained using an energy-dispersive X-ray system (EDS) and a wavelength dispersive spectrometer (WDS) from Oxford Instruments. Orientation imaging microscopy (OIM) was performed using an electron back-scattering diffraction (EBSD) system from HKL Technology.

\section{Results and discussion}

Twinning versus shearing. When the behaviour of CMSX-4 is compared to that of SCA425Hf some similarities are clear but some fundamental differences in deformation mode are observed. Both alloys develop very localized deformation bands which extend across the complete crosssection of the specimen; the fracture surface is purely crystallographic and occurs along one of the $\{111\}$ planes. The main difference is that while twinning is the main deformation mechanism in CMSX-4 during OP-TMF cycling, see figure 1, twinning is only rarely observed in SCA425Hf under similar loading conditions. Instead, shearing is the main deformation mechanism in SCA425Hf, see figure 2. Furthermore, the highly localized deformation in CMSX-4 has been found to promote the precipitation of $\mu$, a topologically close-packed phase (TCP), see figure $1(\mathrm{a})$. This behaviour is rarely observed in SCA425Hf. The difference in deformation mode (twinning versus shearing) is probably attributable to a difference in stacking fault energy (SFE) between the two materials, indicating that SFE is an important parameter for the TMF behaviour in single crystal Nibase superalloys. 

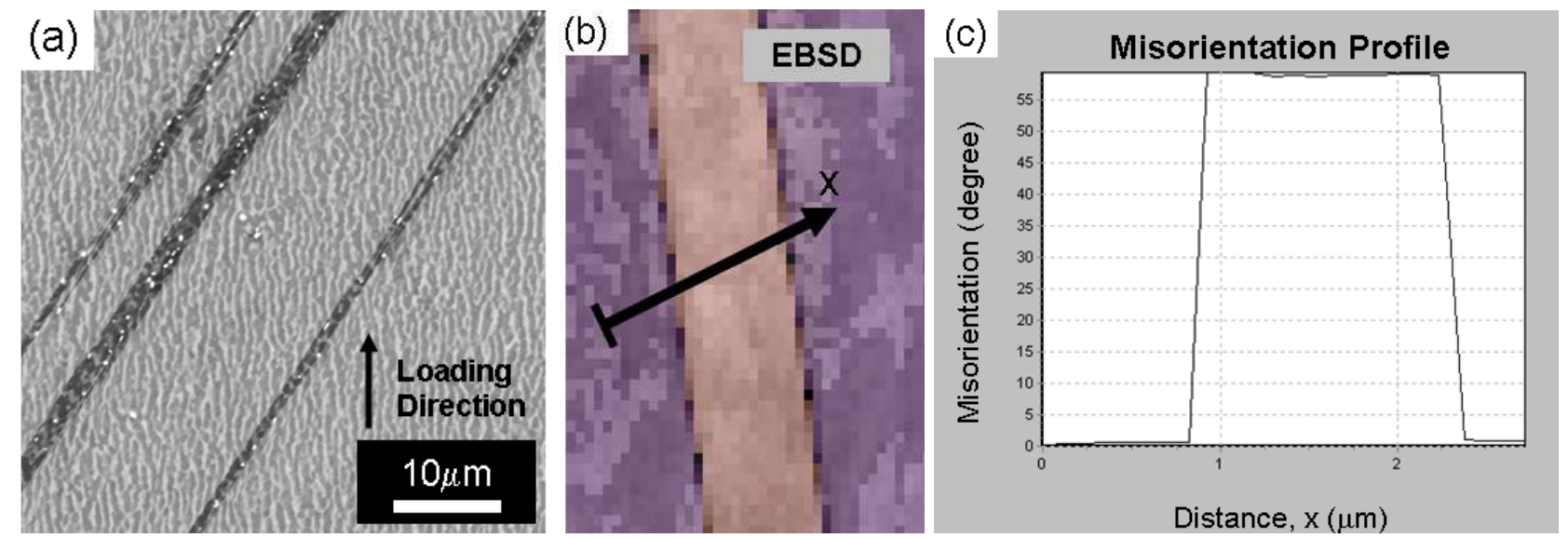

Figure 1: Deformation in CMSX-4 during OP-TMF 100-1000 ${ }^{\circ}$; (a) Backscatter electron micrographs of TMF-damage with localized deformation appearing as twin bands decorated with TCP-precipitates. (b) Crystallographic orientation map produced by EBSD with (c) corresponding misorientation profile transverse to a band of localized deformation giving evidence for twinning.
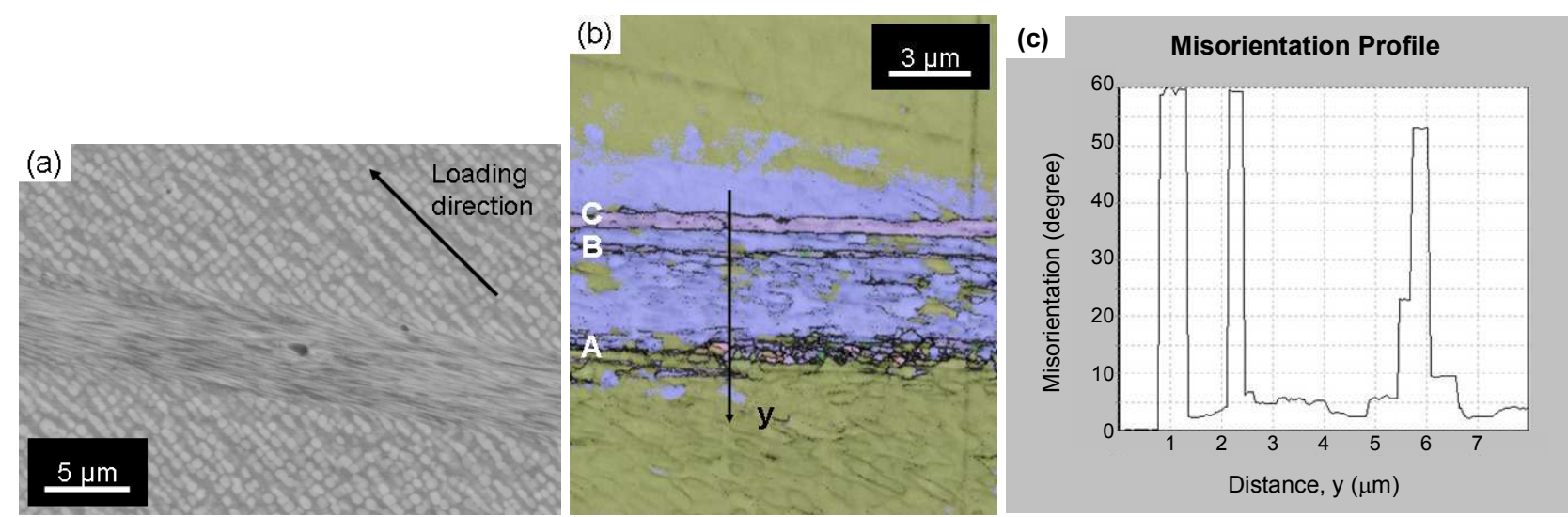

Figure 2: Deformation in SCA425Hf during OP-TMF 100-950 ${ }^{\circ}$; (a) Appearance of the bands of localized deformation. (b) Crystallographic orientation map produced by EBSD showing minor formation of twins and recrystallization. (b) Misorientation profile along the y-axis.

Localized versus dispersed deformation. When CMSX-4 was studied in a long term aged condition, i.e. after 4000 hours of exposure at $1000^{\circ} \mathrm{C}$, it was found that the rather brittle behaviour of virgin single-crystals superalloys (recognized as crystallographic failure along one of the $\{111\}$ planes) under certain conditions could be changed to a rather ductile one [2,3]. This remarkable change in deformation and failure mode between the virgin and long term aged material can be attributed to the material's tendency for the precipitation of TCP-phases. In the virgin material, twinning is an important deformation mode and since the precipitation of TCP-phases for this condition is solely associated with the twins it will promote the tendency for a very localized deformation mode along a few bands extending through the complete cross-section of the specimen. In the aged material, the TCP phases are predominantly formed in the dendritic core regions during the long term ageing; this provides a heterogeneous microstructure which prevents the twins from propagating to a great extent and which consequently promotes a more dispersed deformation, see figure 3(a). The more dispersed mode for the aged material is probably also enhanced by the fact that the TCP precipitates drain the material of some of the most important creep strengthening elements, e.g. Re and W.

Another interesting observation - which still needs a better interpretation - is the crystal rotation (of up to $13^{\circ}$ ) which arises between different regions within the inter-dendritic region, see Figure $3(b, c)$. One can interpret this as an early stage of a misoriented dislocation cell structure and this can only be accomplished by a much more dispersed deformation mode. This type of crystal rotation was not observed in the virgin material after rupture. The observation of crystal rotations in the aged material (as well as the absence of it in the virgin material) has been confirmed by X-ray diffraction measurements. 
These observations demonstrate that the very localized deformation modes often seen in single crystal Ni-base superalloys can be significantly reduced if the alloy possesses a more inhomogeneous microstructure. In fact, several observations indicate that an inhomogeneous microstructure is of great importance for a good TMF resistance. It should be mentioned that even if the low temperature strength and the creep resistance were decreased in the long term aged material, no penalty in the TMF resistance were seen.
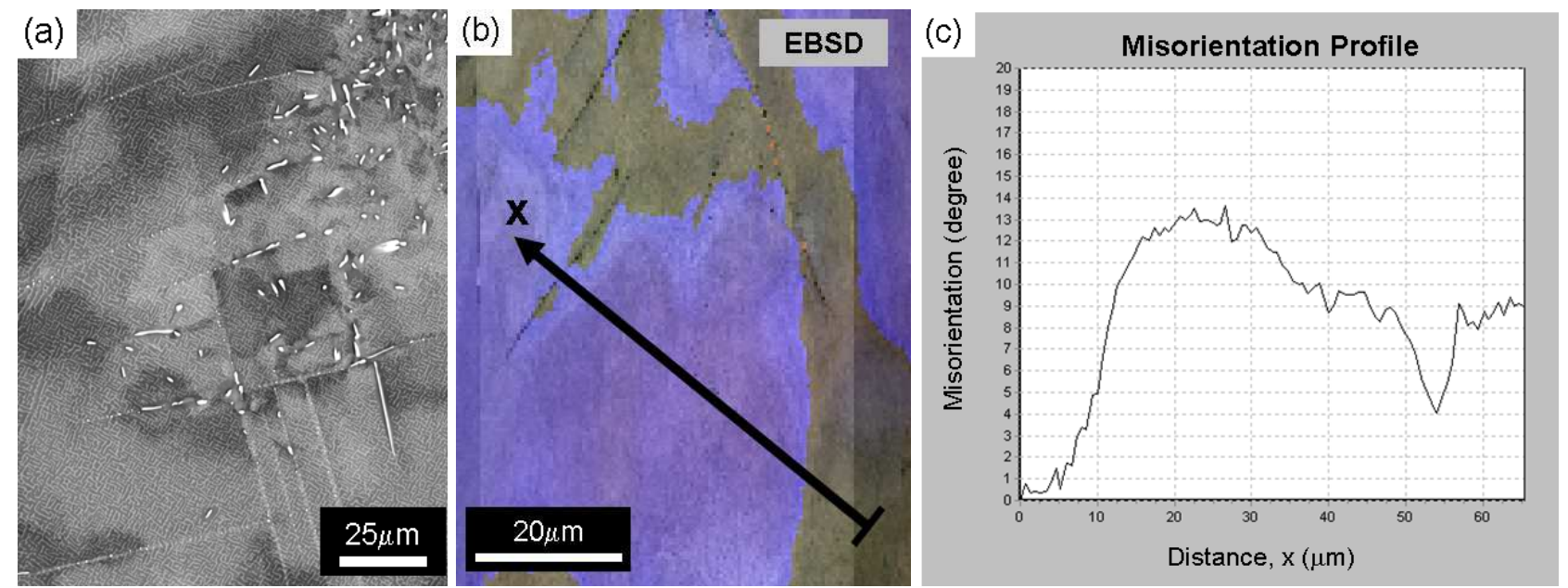

Figure 3: (a) Backscatter electron micrograph of long termed aged CMSX-4 after TMF testing showing twins associated with TCP phases in the dendritic core regions, (b) EBSD map and (c) misorientation profile of the interdendritic region of TMF-tested aged specimen.

Recrystallization. A somewhat remarkable observation from our studies of OP-TMF in single crystal superalloys such as CMSX-4 and SCA425Hf is that recrystallization often occurs during $\mathrm{TMF}$, if the maximum temperature in the test is above $\sim 900^{\circ} \mathrm{C}$. As recrystallized grains also have been observed along the crack front in failed TMF specimens, see figure 4 , it is clear that recrystallization is implicated in the phenomena leading to final failure of the material during TMF.
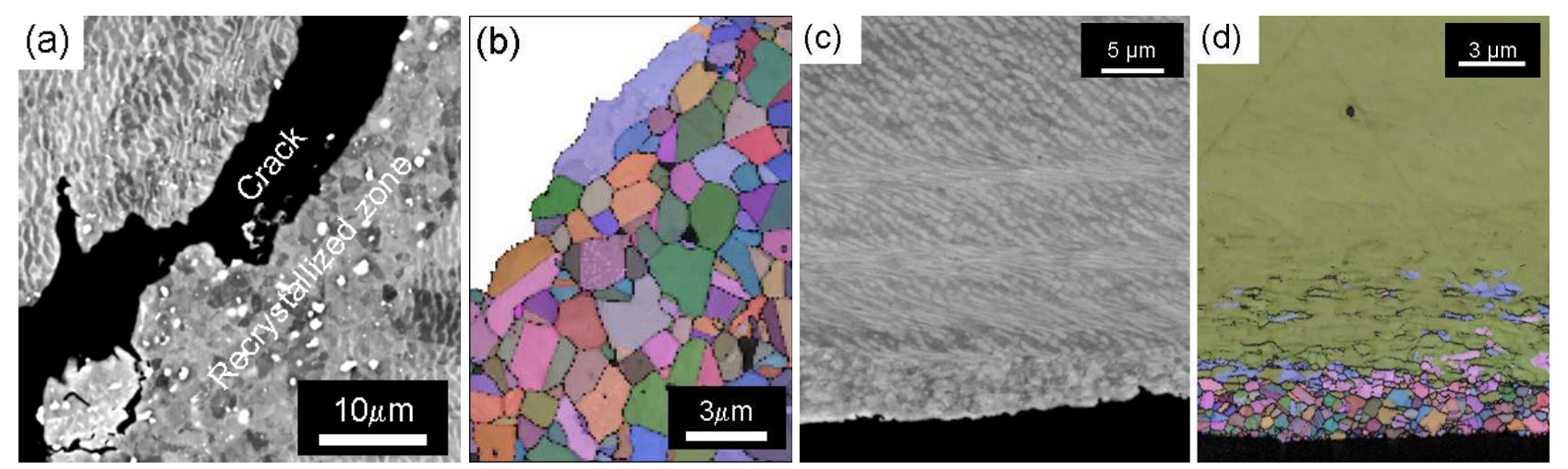

Figure 4: (a) and (b) shows recrystallization in CMSX-4 using backscatter electrons in SEM and EBSD. A crack is running throughout the micrograph. (c) and (d) shows recrystallization in SCA425Hf using backscatter electrons in SEM and EBSD. A fracture surface can be seen in the bottom in the pictures.

Since the absence of grain boundary strengthening elements in these materials is known to lead to premature failure of a recrystallized zone, it is of major interest to understand the details of the recrystallization process occurring under TMF conditions. It is important to realise that the recrystallization is not a consequence of the crack propagation process. Instead, cracking occurs rather easily along the recrystallized bands. In Figure 5, recrystallization has occurred in CMSX-4 without the presence of a crack and one can see that the possible starting point for the 
recrystallization process is the intersection of twins growing in different directions. Once recrystallization has started, it can develop further along the twin directions. Interestingly, no recrystallization is observed for CMSX-4 in the long termed aged condition due to the smaller width and length of the twins and the more dispersed deformation behaviour.
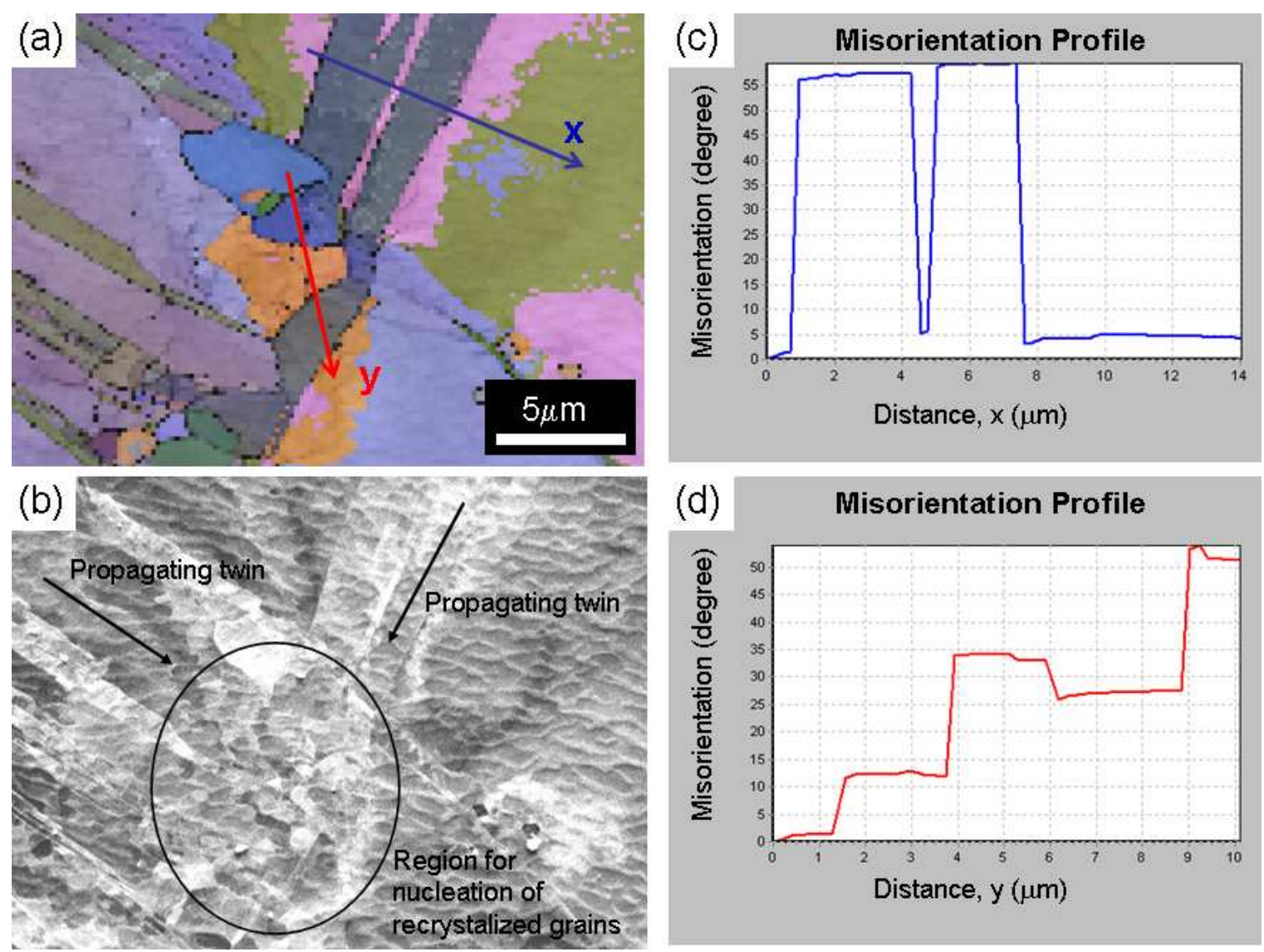

Figure 5: Illustration of the nucleation of recrystallized grains at the intercept of two propagating twins: (a) EBSD map, (b) SEM image. The diagrams (c) and (d) show the crystallographic misorientation along the lines indicated in the EBSD map.

Effect of $\gamma^{\prime}$ size and morphology. Siemens Industrial Turbomachinery have recently initiated a project to find an alloy suitable for industrial gas turbines which exhibits combined high oxidation resistance and high corrosion resistance, see reference [5,6]. As part of this project a modified version of SCA425Hf has been studied which has a higher Al-content; this is currently referred to as SCA425+. The TMF resistance of this alloy has been investigated for different types of heat treatments. The results indicate that the size and morphology of the $\gamma$ ' precipitates can have an effect on the TMF resistance of the alloy. While the creep resistance of the alloy improves with decreasing size of the primary $\gamma$ 'precipitates (approximately $0.35 \mu \mathrm{m}$ and $0.65 \mu \mathrm{m}$, respectively) the opposite influence on the TMF resistance is observed, see figure 6.

In the SCA425+ testpieces one can frequently observe slipping on several slip systems, as seen in figure 7(a), which eventually gives rise to very complex $\gamma^{\prime}$ morphologies as seen in figure 7(b). One also notices that the very elongated shapes of the $\gamma^{\prime}$ phase in the deformation bands are at least partly due to the shearing of the $\gamma^{\prime}$ precipitates, but it is likely that a directional coarsening of the $\gamma^{\prime}$ precipitates (in a similar manner as rafting) is also occurring due to the stress state in the deformation bands. 

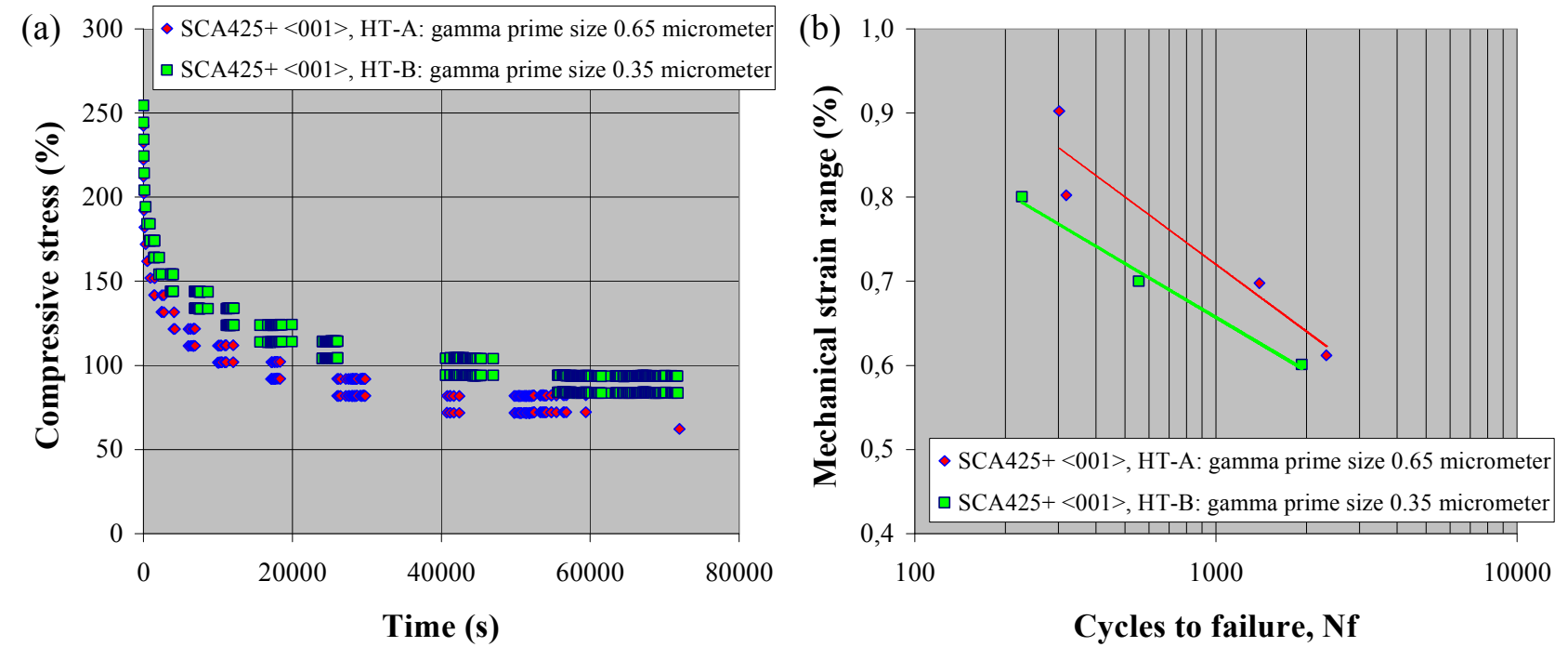

Figure 6: (a) Stress relaxation in $S C A 425+$ during a $20 \mathrm{~h}$ hold time at $950^{\circ} \mathrm{C}$, (b) Mechanical strain range as a function of cycles to failure in SCA425+during OP TMF 100-950 ${ }^{\circ} \mathrm{C}$.
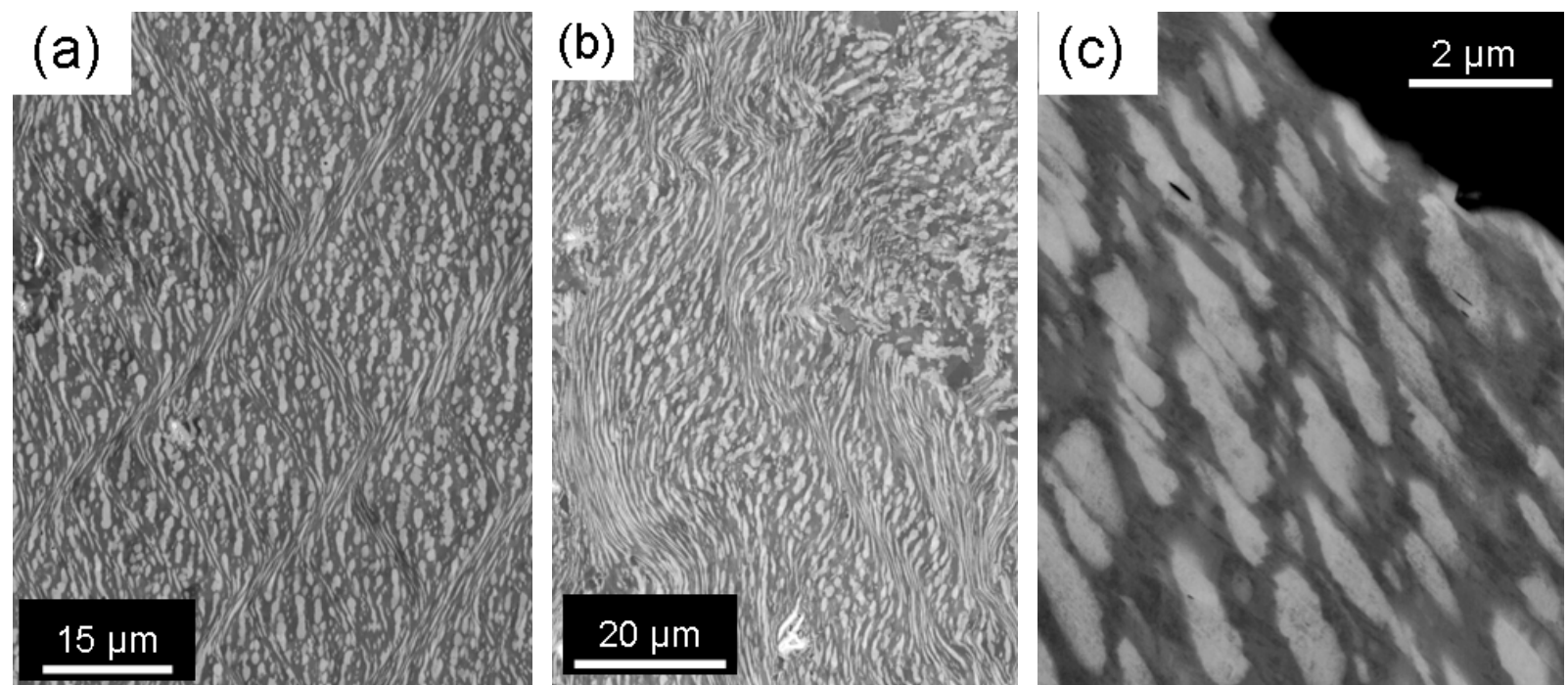

Figure 7: Backscatter electron micrograph of SCA425+ after OP-TMF testing with a maximum temperature of $950^{\circ} \mathrm{C}$. (a-b) Deformation on multiple slip systems. (c) Shearing of $\gamma^{\prime}$ phase

\section{References}

[1] D. Arrell, M. Hasselqvist, C. Sommer and J. Moverare, in: Superalloys 2004, edited by K.A. Green, M. Pollock, H. Harada, T.E. Howson, R.C. Reed, J.J. Schirra, S. Walston, The Minerals, Metals \& Materials Society, Warrendale, PA (2004), p.291

[2] J.J. Moverare, Mater. Sci. Tech. Vol. 23 (2007), p. 1450

[3] J.J. Moverare, S. Johansson and R.C. Reed, Acta Mater. Vol. 57 (2009), p. 2266

[4] J.J. Moverare and S. Johansson, Mater. Sci Eng. A. Vol. 527 (2010), p. 553

[5] A. Sato, J.J. Moverare, M. Hasselqvist and R.C. Reed: On the Oxidation Resistance of NickelBased Superalloys, to be presented at EuroSuperalloys 2010 (this conference)

[6] M. Hasselqvist, R.C. Reed, J.J. Moverare and A. Sato: New Turbine Alloys which Combine Oxidation and Corrosion Resistance, to be presented at EuroSuperalloys 2010 (this conference) 